\title{
Minkowski decompositions of associahedra
}

\author{
Carsten Langeft
}

Freie Universität Berlin, FB Mathematik und Informatik, Berlin, Germany

\begin{abstract}
Realisations of associahedra can be obtained from the classical permutahedron by removing some of its facets and the set of facets is determined by the diagonals of certain labeled convex planar $n$-gons as shown by Hohlweg and Lange (2007). Ardila, Benedetti, and Doker (2010) expressed polytopes of this type as Minkowski sums and differences of scaled faces of a standard simplex and computed the corresponding coefficients $y_{I}$ by Möbius inversion from the $z_{I}$ if tight right-hand sides $z_{I}$ for all inequalities of the permutahedron are assumed. Given an associahedron of Hohlweg and Lange, we first characterise all tight values $z_{I}$ in terms of non-crossing diagonals of the associated labeled $n$-gon, simplify the formula of Ardila et al., and characterise the remaining terms combinatorially.

Résumé. Dans un article paru en 2007, Hohlweg et Lange décrivent des associaèdres réalisés à partir du permutoédre en enlevant certaines de ses facettes. Ces facettes sont déterminées par les diagonales d'une famille de $n$-gones étiquetés. En 2010, Ardila, Benedetti et Doker ont montré que ces polytopes s'expriment par des sommes et différences de Minkowski de faces pondérées d'un simplexe. De plus, si les coefficients $z_{I}$ des inégalités décrivant l'associaèdre à partir du permutoédre sont optimaux, alors les coefficients $y_{I}$ correspondants sont calculés par une inversion de Möbius. Étant donné un tel associaèdre, nous décrivons d'abord les valeurs optimales $z_{I}$ en termes de diagonales non croisées d'un certain $n$-gone étiqueté, ensuite nous simplifions la formule de Ardila et al. pour finalement décrire combinatoirement les termes restants.
\end{abstract}

Keywords: reflection and Coxeter groups, lattice polytopes, associahedra, Minkowski sums

\section{Generalised permutahedra and associahedra}

Generalised permutahedra and Minkowski decompositions. A generalised permutahedron according to A. Postnikov is a convex $(n-1)$-polytope that has the following description by inequalities

$$
P_{n}\left(\left\{z_{I}\right\}\right):=\left\{\boldsymbol{x} \in \mathbb{R}^{n} \mid \sum_{i \in[n]} x_{i}=z_{[n]} \text { and } \sum_{i \in I} x_{i} \geq z_{I} \text { for } \varnothing \subset I \subset[n]\right\},
$$

where $[n]$ denotes the set $\{1,2, \cdots, n\}$, [Pos09]. The classical permutahedron, as described for example by G. M. Ziegler, [Zie98], corresponds to the choice $z_{I}=\frac{|I|(|I|+1)}{2}$ for $\varnothing \subset I \subseteq[n]$. Obviously, some of the above inequalities may be redundant for $P_{n}\left(\left\{z_{I}\right\}\right)$ and sufficiently small increases and decreases of $z_{I}$ of redundant inequalities do not change the set $P_{n}\left(\left\{z_{I}\right\}\right)$ unless the inequality $\sum_{i \in I} x_{i} \geq z_{I}$ is tight. As described next, all generalised permutahedra have a Minkowski decomposition into faces of a simplex and the coefficients $y_{I}$ of this decomposition can be computed if all (tight) values $z_{I}$ are known.

\footnotetext{
${ }^{\dagger}$ Partially supported by the DFG grant (Forschergruppe 565 Polyhedral Surfaces). 
For any two polytopes $P$ and $Q$, the Minkowski sum $P+Q$ is defined as $\{p+q \mid p \in P, q \in Q\}$. In contrast, we define the Minkowski difference $P-Q$ of $P$ and $Q$ only if there is a polytope $R$ such that $P=Q+R$. We are interested in decompositions of generalised permutahedra into Minkowski sums and differences of faces of the $(n-1)$-dimensional standard simplex

$$
\Delta_{n}=\operatorname{conv}\left\{e_{1}, e_{2}, \cdots, e_{n}\right\},
$$

where $e_{i}$ is a standard basis vector of $\mathbb{R}^{n}$. The faces $\Delta_{I}$ of $\Delta_{n}$ are given by $\operatorname{conv}\left\{e_{i}\right\}_{i \in I}$ for $I \subseteq[n]$. If a polytope $P$ is the Minkowski sum and difference of scaled faces of $\Delta_{n}$, we say that $P$ has a Minkowski decomposition into faces of the standard simplex. The following two results are key observations.

Lemma 1.1 (|ABD10, Lemma 2.1]) $P_{n}\left(\left\{z_{I}\right\}\right)+P_{n}\left(\left\{z_{I}^{\prime}\right\}\right)=P_{n}\left(\left\{z_{I}+z_{I}^{\prime}\right\}\right)$.

If we consider the function $I \longmapsto z_{I}$ that assigns every subset of $[n]$ the corresponding tight value $z_{I}$ of $P_{n}\left(\left\{z_{I}\right\}\right)$, then the Möbius inverse of this function assigns to $I$ the coefficient $y_{I}$ of a Minkowski decomposition of $P_{n}\left(\left\{z_{I}\right\}\right)$ into faces of the standard simplex:

Proposition 1.2 ([ABD10, Proposition 2.3])

Every generalised permutahedron $P_{n}\left(\left\{z_{I}\right\}\right)$ can be written uniquely as a Minkowski sum and difference offaces of $\Delta_{n}$ :

$$
P_{n}\left(\left\{z_{I}\right\}\right)=\sum_{I \subseteq[n]} y_{I} \Delta_{I}
$$

where $y_{I}=\sum_{J \subseteq I}(-1)^{|I \backslash J|} z_{J}$ for each $I \subseteq[n]$.

Studying properties of generalised permutahedra, Postnikov proved a weaker version of Proposition 1.2 that requires $y_{I} \geq 0$ for all $I \subseteq[n]$, [Pos09]. Although we can compute the values $y_{I}$ theoretically if all tight values $z_{I}$ are known, the formula of Proposition 1.2 is computationally expensive.

We could stop here and be fascinated how the Möbius inversion relates the description by half spaces and Minkowski decompositions. Nevertheless, we go further and study this relationship for certain realisations of associahedra, a certain subclass of generalised permutahedra described in the following subsection. It turns out that the formula of Ardila, Benedetti \& Doker can be simplified significantly and that there is a combinatorial interpretation of the terms that remain after this simplification. The simplified formula extracts the combinatorial core data for the Möbius inversion: which subsets $J$ of $I$ are essential to compute $y_{I}$. In general, the sets $J$ obtained for the same $I$ but different realisations of an $n$-dimensional associahedron are different. Nevertheless, the sets $J$ have a simple combinatorial characterisation as shown in Section 3

Associahedra as generalised permutahedra. Associahedra form a class of combinatorially equivalent simple polytopes and can be realised as generalised permutahedra. They are often defined by specifying their 1-skeleton or graph. A theorem of G. Kalai, [Kal88], implies that the face lattice of an $(n-1)$ dimensional associahedron $\mathrm{As}_{n-1}$ is in fact completely determined by this graph. Now, the graph of an associahedron is isomorphic to a graph with all triangulations (without new vertices) of a convex and plane $(n+2)$-gon $Q$ as vertex set and all pairs of distinct triangulations that differ in precisely one proper diagona $\left({ }^{(i)}\right]$ as edge set. Alternatively, the edges of $A s_{n-1}$ are in bijection with the set of triangulations with

(i) A proper diagonal is a line segment connecting a pair of vertices of $Q$ whose relative interior is contained in the interior of $Q$. A non-proper diagonal is a diagonal that connects vertices adjacent in $\partial Q$ and a degenerate diagonal is a diagonal where the end-points are equal. 
one proper diagonal removed. Similarly, $k$-faces of $\mathrm{As}_{n-1}$ are in bijection to triangulations of $Q$ with $k$ proper diagonals deleted. In particular, the facets of $A s_{n-1}$ are in bijection with the proper diagonals of $Q$. J.-L. Loday published a beautiful construction of associahedra in 2004, [Lod04]. This construction was generalised by C. Hohlweg and C. Lange, [HL07], and explicitly describes realisations of $A s_{n-1}$ as generalised permutahedra that depend on combinatorics induced by the choice of a Coxeter element $c$ of the symmetric group $\Sigma_{n}$ on $n$ elements. Figure 1 shows two realisations of $\mathrm{As}_{3}$ for different choices of $c$. Before explaining how to obtain these realisations, we stop for some general remarks.

S. Fomin and A. Zelevinsky introduced generalised associahedra in the context of cluster algebras of finite type, [FZ03], and it is well-known that associahedra and generalised associahedra associated to cluster algebras of type $A$ are combinatorially equivalent. The construction of [HL07] was generalised by C. Hohlweg, C. Lange, and H. Thomas, [HLT11] to generalised associahedra. Their construction depends also on choosing a Coxeter element $c$ and the normal vectors of the facets are determined by combinatorial properties of $c$. Since the normal fans of these realisations turn out to be Cambrian fans as described by N. Reading and D. Speyer, [RS09], the obtained realisations are generalised associahedra associated to any given cluster algebra of finite type. N. Reading and D. Speyer conjectured a linear isomorphism between Cambrian fans and $g$-vector fans associated to cluster algebras of finite type with acyclic initial seed introduced by S. Fomin and A. Zelevinsky, [FZ07]. They proved their conjecture up to an assumption of another conjecture of [FZ07]. S.-W. Yang and A. Zelevinsky gave an alternative proof of the conjecture of Reading and Speyer in [YZ08]. We emphazise in this context that the results of Section 2 and 3 can be read along these lines: the computations of $z_{I}$ and $y_{I}$ for fixed $I$ and varying $c$ involve sums over different choices of $z_{R_{\delta}}$ where the choice of $\delta$ depends on $c$. Moreover, the values $z_{R_{\delta}}$ that occur in these sums can be choosen within a large class as described for example in [HLT11]. From this point of view, we suggest that combinatorial properties of the $g$-vector fan for cluster algebras of finite type $A$ with respect to an acyclic initial seed are reflected by the Minkowski decompositions studied in this manuscript.

We now return to the construction of [HL07] and give a brief outline. The choice of a Coxeter element $c$ corresponds to a partition of $[n]$ into a down set $\mathrm{D}_{c}$ and an up set $\mathrm{U}_{c}$ :

$$
\mathrm{D}_{c}=\left\{d_{1}=1<d_{2}<\cdots<d_{\ell}=n\right\} \quad \text { and } \quad \mathrm{U}_{c}=\left\{u_{1}<u_{2}<\cdots<u_{m}\right\} .
$$

This partition induces a labeling of $Q$ with label set $[n+1]_{0}:=[n+1] \cup\{0\}$ as follows. Pick two vertices of $Q$ which are the end-points of a path with $\ell+2$ vertices on the boundary of $Q$, label the vertices of this path counter-clockwise increasing using the label set $\overline{\mathrm{D}}_{c}:=\mathrm{D}_{c} \cup\{0, n+1\}$ and label the remaining path clockwise increasing using the label set $\mathrm{U}_{c}$. Without loss of generality, we shall always assume that the label set $\mathrm{D}_{c}$ is on the right-hand side of the diagonal $\{0, n+1\}$ oriented form 0 to $n+1$, see Figure 1 for two examples. We derive the values $z_{I}$ for some subsets $I \subset[n]$ obtained from this labeled $(n+2)$-gon $Q$ using proper diagonals of $Q$ as follows. Orient each proper diagonal $\delta$ from the smaller to the larger labeled end-point of $\delta$, associate to $\delta$ the set $R_{\delta}$ that consists of all labels on the strict right-hand side of $\delta$, and replace the elements 0 and $n+1$ by the smaller respectively larger label of the end-points contained in $\mathrm{U}_{c}$ if possible. For each proper diagonal $\delta$ we have $R_{\delta} \subseteq[n]$ but for $n>2$, obviously not every subset of $[n]$ is of this type. We set

$$
\tilde{z}_{I}^{c}:= \begin{cases}\frac{|I|(|I|+1)}{2} & \text { if } I=R_{\delta} \text { for some proper diagonal } \delta, \\ -\infty & \text { else, }\end{cases}
$$

compare Table 1 for the examples of Figure 1 In [HL07] it is shown that $P_{n}\left(\left\{\tilde{z}_{I}^{c}\right\}\right)$ is in fact an associa- 

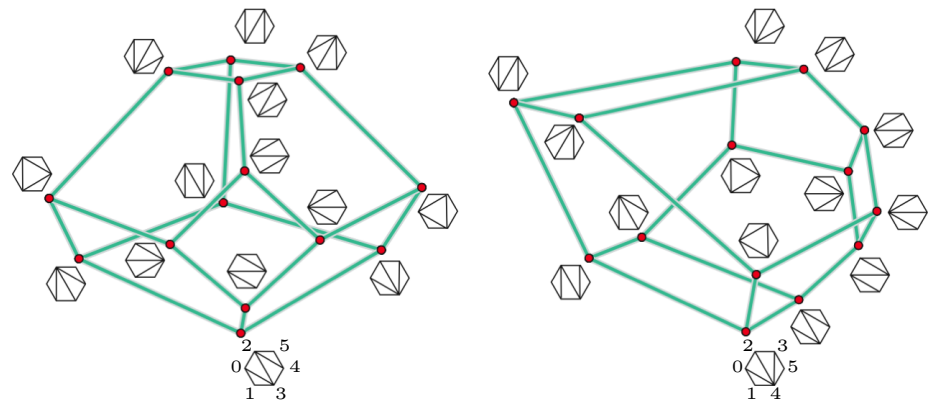

Fig. 1: Two 3-dimensional associahedra $A s_{3}^{c}=P_{4}\left(\left\{\tilde{z}_{I}^{c}\right\}\right)$ with vertex coordinates computed for differently choosen Coxeter elements according to [HL07] after application of an orthogonal transformation. The different Coxeter elements are encoded by different labelings of hexagons as indicated.

$\mathrm{D}_{c}=\{1,3,4\}$ and $\mathrm{U}_{c}=\{2\}:$

\begin{tabular}{l|r|r|r|r|r|r|r|r|r}
$\delta$ & $\{0,3\}$ & $\{0,4\}$ & $\{0,5\}$ & $\{1,2\}$ & $\{1,4\}$ & $\{1,5\}$ & $\{2,3\}$ & $\{2,4\}$ & $\{3,5\}$ \\
\hline$R_{\delta}$ & $\{1\}$ & $\{1,3\}$ & $\{1,3,4\}$ & $\{2,3,4\}$ & $\{3\}$ & $\{3,4\}$ & $\{1,2\}$ & $\{1,2,3\}$ & $\{4\}$ \\
\hline$\tilde{z}_{R_{\delta}}$ & 1 & 3 & 6 & 6 & 1 & 3 & 3 & 6 & 1
\end{tabular}

$\mathrm{D}_{c}=\{1,4\}$ and $\mathrm{U}_{c}=\{2,3\}$ :

\begin{tabular}{l|r|r|r|r|r|r|r|r|r}
$\delta$ & $\{0,4\}$ & $\{2,4\}$ & $\{3,4\}$ & $\{0,5\}$ & $\{0,3\}$ & $\{1,2\}$ & $\{2,5\}$ & $\{1,3\}$ & $\{1,5\}$ \\
\hline$R_{\delta}$ & $\{1\}$ & $\{1,2\}$ & $\{1,2,3\}$ & $\{1,4\}$ & $\{1,3,4\}$ & $\{2,3,4\}$ & $\{1,2,4\}$ & $\{3,4\}$ & $\{4\}$ \\
\hline$\tilde{z}_{R_{\delta}}$ & 1 & 3 & 6 & 3 & 6 & 6 & 6 & 3 & 1
\end{tabular}

Tab. 1: The tables list $R_{\delta}$ and $z_{I}$ associated to the proper diagonal $\delta$ of a labeled hexagon. The upper table corresponds to the associahedron shown on the left of Figure 1 the bottom one to the one on the right. 
hedron of dimension $n-1$ realised in $\mathbb{R}^{n}$ for every choice of $c$. We refer to these realisations as $\mathrm{As}_{n-1}^{c}$. As shown by C. Hohlweg, C. Lange, and H. Thomas, one also obtains associahedra if the finite values of $\tilde{z}_{I}^{c}$ are replaced by the corresponding right-hand sides of a permutahedron obtained as convex hull of a (non-degenerate) $\Sigma_{n}$-orbit of a point of $\mathbb{R}^{n}$, where $\Sigma_{n}$ acts by permutation of coordinates, [HLT11]. The results of Sections 2 and 3 remain true in this situation.

Some instances of $A s_{n-1}^{c}$ have been studied earlier. For example, the realisations of J.-L. Loday, [Lod04], and of G. Rote, F. Santos, and I. Streinu, [RSS03], related to one-dimensional point configurations, are affinely equivalent to $\mathrm{As}_{n-1}^{c}$ if $\mathrm{U}_{c}=\varnothing$ or $\mathrm{U}_{c}=\{2,3\}$. Moreover, G. Rote, F. Santos, and I. Streinu point out in Section 5.3 that their realisation is not affinely equivalent to the realisation of F. Chapoton, S. Fomin, and A. Zelevinsky, [CFZ02], which in turn is affinely equivalent to $\mathrm{As}_{n-1}^{c}$ if $\mathrm{U}_{c}=\{2\}$ or $\mathrm{U}_{c}=\{3\}$.

Outline. In this article, we study Minkowski decompositions of a family of realisations of associahedra. In the next section, we explain a combinatorial procedure to compute tight values $z_{I}$ for the redundant inequalities from the irredundant ones for the realisations of [HL07], compare Theorem 2.8. A key step for the computation is the up and down interval decomposition of $I$ defined in Definition 2.2. In Section 3 , we simplify the formula for $y_{I}$ of Proposition 1.2 and show that most terms in that alternating sum cancel. It turns out that at most four summands remain and they are combinatorially characterised by properties of the up and down interval decomposition of $I$, see Theorem 3.1 for the precise statement. A full version of this extended abstract that includes all proofs will be published later.

\section{Tight values for all $z_{I}^{c}$ for $\mathrm{As}_{n-1}^{c}$}

As already mentioned, the facet-defining inequalities for $\mathrm{As}_{n-1}^{c}$ correspond to proper diagonals of $Q$ and these are precisely the irredundant inequalities for the generalised permutahedron $P_{n}\left(\left\{\tilde{z}_{I}^{c}\right\}\right)$. As motivated in the previous section, we want to determine tight values for all $z_{I}, I \subseteq[n]$, in order to compute the coefficients $y_{I}$ of the Minkowski decomposition of $\mathrm{As}_{n-1}^{c}$ described by F. Ardila, C. Benedetti, and J. Doker. Since we already know that $\tilde{z}_{I}^{c}$ is a tight value if and only if $I=R_{\delta}$ for some proper diagonal of $Q$, we now aim for a description of the missing $\tilde{z}_{I}^{c}$. The concept of an up and down interval decomposition induced by the partitioning $\mathrm{D}_{c} \cup \mathrm{U}_{c}$ (or equivalently induced by $c$ ) of a given interval $I \subset[n]$ is a key concept that we introduce first.

\section{Definition 2.1 (up and down intervals)}

Let $\mathrm{D}_{c}=\left\{d_{1}=1<d_{2}<\cdots<d_{\ell}=n\right\}$ and $\mathrm{U}_{c}=\left\{u_{1}<u_{2}<\cdots<u_{m}\right\}$ be the partition of $[n]$ induced by a Coxeter element $c$.

(a) A set $S \subseteq[n]$ is a non-empty interval of $[n]$ if $S=\{r, r+1, \cdots, s\}$ for some $0<r \leq s<n$. We write $S$ as closed interval $[r, s]$ (end-points included) or as open interval $(r-1, s+1)$ (end-points not included). An empty interval is an open interval $(k, k+1)$ for some $1 \leq k<n$.

(b) A non-empty open down interval is a set $S \subseteq \mathrm{D}_{c}$ such that $S=\left\{d_{r}<d_{r+1}<\cdots<d_{s}\right\}$ for some $1 \leq r \leq s \leq \ell$. We write $S$ as open down interval $\left(d_{r-1}, d_{s+1}\right)_{\mathrm{D}_{c}}$ where we allow $d_{r-1}=0$ and $d_{s+1}=n+1$, i.e. $d_{r-1}, d_{s+1} \in \overline{\mathrm{D}}_{c}$. For $1 \leq r \leq \ell-1$, we have the empty down inter$\operatorname{val}\left(d_{r}, d_{r+1}\right) \mathrm{D}_{c}$.

(c) A closed up interval is a non-empty set $S \subseteq \mathrm{U}_{c}$ such that $S=\left\{u_{r}<u_{r+1}<\cdots<u_{s}\right\}$ for some $1 \leq r \leq s \leq \ell$. We write $\left[u_{r}, u_{s}\right] \mathrm{U}_{c}$. 
We often omit the words open and closed when we consider down and up intervals. There will not be any ambiguity, because we are not going to deal with closed down intervals and open up intervals. Up intervals are always non-empty, while down intervals may be empty. It turns out to be convenient to distinguish the empty down intervals $\left(d_{r}, d_{r+1}\right)_{\mathrm{D}_{c}}$ and $\left(d_{s}, d_{s+1}\right)_{\mathrm{D}_{c}}$ if $r \neq s$ although they are equal as sets.

\section{Definition 2.2 (up and down interval decomposition)}

Let $\mathrm{D}_{c}=\left\{d_{1}=1<d_{2}<\cdots<d_{\ell}=n\right\}$ and $\mathrm{U}_{c}=\left\{u_{1}<u_{2}<\cdots<u_{m}\right\}$ be the partition of $[n]$ induced by a Coxeter element $c$ and $I \subseteq[n]$ be non-empty.

(a) An up and down interval decomposition of type $(v, w)$ of $I$ is a partition of I into disjoint up and down intervals $I_{1}^{\mathrm{U}}, \cdots, I_{w}^{\mathrm{U}}$ and $I_{1}^{\mathrm{D}}, \cdots, I_{v}^{\mathrm{D}}$ obtained by the following procedure.

1. Suppose there are $\tilde{v}$ non-empty inclusion maximal down intervals of I denoted by $\tilde{I}_{k}^{\mathrm{D}}=\left(\tilde{a}_{k}, \tilde{b}_{k}\right)_{\mathrm{D}_{c}}$, $1 \leq k \leq \tilde{v}$, with $\tilde{b}_{k} \leq \tilde{a}_{k+1}$ for $1 \leq k<\tilde{v}$. Let $E_{i}^{\mathrm{D}}=\left(d_{r_{i}}, d_{r_{i}+1}\right)_{\mathrm{D}_{c}}$ denote all empty down intervals with $\tilde{b}_{k} \leq d_{r_{i}}<d_{r_{i}+1} \leq \tilde{a}_{k+1}$ for $0 \leq k \leq \tilde{v}$, $\tilde{b}_{0}=0$, and $\tilde{a}_{\tilde{v}+1}=n+1$. Denote the open intervals $\left(\tilde{a}_{i}, \tilde{b}_{i}\right)$ and $\left(d_{r_{i}}, d_{r_{i}+1}\right)$ of $[n]$ by $\tilde{I}_{i}$ and $E_{i}$ respectively.

2. Consider all up intervals of $I$ which are contained in (and inclusion maximal within) some interval $\tilde{I}_{i}$ or $E_{i}$ obtained in Step 1 and denote these up intervals by

$$
I_{1}^{\cup}=\left[\alpha_{1}, \beta_{1}\right]_{\cup_{c}}, \cdots, I_{w}^{\cup}=\left[\alpha_{w}, \beta_{w}\right]_{\cup_{c}} .
$$

Without loss of generality, we assume $\alpha_{i} \leq \beta_{i}<\alpha_{i+1}$.

3. A down interval $I_{i}^{\mathrm{D}}=\left(a_{i}, b_{i}\right)_{\mathrm{D}_{c}}, 1 \leq i \leq w$, is a down interval obtained in Step 1 that is either a non-empty down interval $\tilde{I}_{k}^{\mathrm{D}}$ or an empty down interval $E_{k}^{\mathrm{D}}$ with the additional property that there is some up interval $I_{j}^{\mathrm{U}}$ obtained in Step 2 such that $I_{j}^{\mathrm{U}} \subseteq E_{k}$. Without loss of generality, we assume $b_{i} \leq a_{i+1}$ for $1 \leq i<w$.

(b) An up and down interval decomposition of type $(1, w)$ is called nested. A nested component of $I$ is an inclusion-maximal subset $J$ of I such that the up and down decomposition of $J$ is nested.

The following example illustrates Lemma 2.4 and Lemma 2.5 .

\section{Example 2.3}

Let $\mathrm{D}_{c}=\left\{d_{1}=1<d_{2}<\cdots<d_{\ell}=n\right\}$ and $\mathrm{U}_{c}=\left\{u_{1}<u_{2}<\cdots<u_{m}\right\}$ be the partition of $[n]$ induced by a Coxeter element $c$. The proper diagonals $\delta=\{a, b\}, a<b$, of the labeled polygon $Q$ are in bijection to certain non-empty proper subsets $I \subset[n]$ that have an up and down interval decomposition of type $(1,0),(1,1)$, or $(1,2)$. More precisely, we have

(a) $R_{\delta}=(a, b)_{\mathrm{D}_{c}}$ if and only if $R_{\delta}$ has an up and down decomposition of type $(1,0)$.

(b) $R_{\delta}=(0, b)_{\mathrm{D}_{c}} \cup\left[u_{1}, a\right]_{\cup_{c}}$ or $R_{\delta}=(a, n+1)_{\mathrm{D}_{c}} \cup\left[b, u_{m}\right]_{\cup_{c}}$ if and only if $R_{\delta}$ has a decomposition of type $(1,1)$.

(c) $R_{\delta}=(0, n+1)_{\mathrm{D}_{c}} \cup\left[u_{1}, a\right]_{\cup_{c}} \cup\left[b, u_{m}\right]_{U p_{c}}$ if and only if $R_{\delta}$ has an up and down decomposition of type $(1,2)$.

\section{Lemma 2.4}

Given a partition $[n]=\mathrm{D}_{c} \sqcup \mathrm{U}_{c}$ induced by a Coxeter element $c$ and a non-empty subset $I \subseteq[n]$. Let I have a nested up and down interval decomposition of type $(1,0)$ with down interval $(a, b)_{\mathrm{D}_{c}}$ and no up interval $\left[\alpha_{i}, \beta_{i}\right]_{\mathrm{U}_{c}}$. Associate the diagonal $\delta_{1}:=\{a, b\}$ to $I$.

(a) The diagonal $\delta_{1}$ is a proper diagonal if and only if $I \subset[n]$.

(b) If the diagonal $\delta_{1}$ is not proper then $\delta_{1}=\{0, n\}$, in particular $\mathrm{U}_{c}=\varnothing$. 
(c) $I=R_{\delta_{1}}$.

Lemma 2.5

Given a partition $[n]=\mathrm{D}_{c} \sqcup \mathrm{U}_{c}$ induced by the Coxeter element $c$ and a non-empty subset $I \subseteq[n]$. Let $I$ have a nested up and down interval decomposition of type $(1, w)$ with $w \geq 1$, down interval $(a, b)_{\mathrm{D}_{c}}$, and up intervals $\left[\alpha_{i}, \beta_{i}\right]_{\mathrm{U}_{c}}$ for $1 \leq i \leq w$. Associate $w+1$ diagonals $\delta_{i}$ to $I$ :

$$
\delta_{1}:=\left\{a, \alpha_{1}\right\}, \quad \delta_{i}:=\left\{\beta_{i-1}, \alpha_{i}\right\} \text { for } 1<i<w+1, \text { and } \quad \delta_{w+1}:=\left\{\beta_{w}, b\right\} .
$$

Then

(a) The diagonals $\delta_{i}$ are proper diagonals of $Q$ for $1<i<w+1$.

(b) The diagonal $\delta_{1}$ is either a proper diagonal or the edge $\left\{0, u_{1}\right\}$ of $Q$.

(c) The diagonal $\delta_{w+1}$ is either a proper diagonal or the edge $\left\{u_{m}, n+1\right\}$ of $Q$.

(d) The diagonals $\delta_{i}$ are non-crossing.

(e) Let $W \subseteq[w+1]$ be the index set of the proper diagonals among $\delta_{1}, \cdots, \delta_{w+1}$ and $m$ be the maximal element of $W$. Then

$$
I=R_{\delta_{m}} \backslash\left(\bigcup_{i \in W \backslash\{m\}}[n] \backslash R_{\delta_{i}}\right) .
$$

Proof: We only prove statement $(e)$. The up and down interval decomposition of $I$ guarantees $I \subseteq R_{\delta_{i}}$ for $i \in W$. Thus

$$
R_{\delta_{m}} \cap R_{\delta_{i}}=R_{\delta_{m}} \backslash\left([n] \backslash R_{\delta_{i}}\right), \quad \text { for } i \in W \backslash\{m\}
$$

and

$$
I=\bigcap_{i \in W} R_{\delta_{i}}=\bigcap_{i \in W \backslash\{m\}}\left(R_{\delta_{m}} \cap R_{\delta_{i}}\right)=R_{\delta_{m}} \backslash\left(\bigcup_{i \in W \backslash\{m\}}[n] \backslash R_{\delta_{i}}\right) .
$$

\section{Lemma 2.6}

Given a partition $[n]=\mathrm{D}_{c} \sqcup \mathrm{U}_{c}$ induced by a Coxeter element $c$. Let I be a non-empty proper subset of $[n]$ with up and down interval decomposition of type $(v, w)$. Then there are $v$ nested components of type $\left(1, w_{1}\right), \cdots,\left(1, w_{v}\right)$ with $w=\sum_{j=1}^{v} w_{j}$. For $1 \leq i \leq v$ and $1 \leq j \leq w_{i}$ let $\left[\alpha_{i, j}, \beta_{i, j}\right]_{\mathrm{U}_{c}}$ denote the up intervals nested in the down interval $\left(a_{i}, b_{i}\right)_{\mathrm{D}_{c}}$ and associate $w_{i}+1$ diagonals $\delta_{i, j}, 1 \leq j \leq w_{i}+1$, to each nested component in the same way as in Lemma 2.4 and Proposition 2.5. Then

(a) The diagonals $\delta_{i, j}$ are non-crossing.

(b) As in Proposition 2.5 it can happen that we have to adjust to index sets $W_{1}$ and $W_{v}$ to avoid nonproper diagonals.

$$
I=\bigcup_{i=1}^{v}\left(R_{\delta_{i, m_{i}}} \backslash\left(\bigcup_{j \in W_{i} \backslash\left\{m_{i}\right\}}[n] \backslash R_{\delta_{i, j}}\right)\right) .
$$

Proof: There are precisely $v$ nested components, one for every down interval. Moreover, for each up interval $[\alpha, \beta]_{\mathrm{U}_{c}}$ exists a unique down interval $(a, b)_{\mathrm{D}_{c}}$ with $[\alpha, \beta]_{\mathrm{U}_{c}} \subseteq(a, b)$. Depending on the type, apply Lemma 2.4 or Lemma 2.5 to each nested component. Combining the statements gives the claim. 
Definition 2.7 Given a partition $[n]=\mathrm{D}_{c} \sqcup \mathrm{U}_{c}$ induced by a Coxeter element $c$. Let I be a nonempty proper subset of $[n]$ with up and down interval decomposition of type $(v, w)$, nested components of type $\left(1, w_{1}\right), \cdots,\left(1, w_{v}\right)$ and associated diagonals $\delta_{i, j}$. The subset $\mathcal{D}_{I}$ of proper diagonals of $\left\{\delta_{i, j} \mid 1 \leq i \leq v\right.$ and $\left.1 \leq j\right\}$ is called set of proper diagonals associated to $I$. Similarly, we say that $\delta \in \mathcal{D}_{I}$ is a proper diagonal associated to $I$.

\section{Theorem 2.8}

Given a partition $[n]=\mathrm{D}_{c} \sqcup \mathrm{U}_{c}$ induced by a Coxeter element $c$. Let I be a non-empty proper subset of $[n]$ with up and down interval decomposition of type $(v, w)$. We use the notation of Lemma 2.6 for its up and down interval decomposition. For non-empty $I \subseteq[n]$ we set

$$
z_{I}^{c}:=\sum_{i=1}^{v}\left(\sum_{j \in W_{i}} \tilde{z}_{R_{\delta_{i, j}}^{c}}-\left(\left|W_{i}\right|-1\right) z_{[n]}\right) .
$$

Then $P\left(\left\{z_{I}^{c}\right\}\right)=P\left(\left\{\tilde{z}_{I}^{c}\right\}\right)=\mathrm{As}_{n-1}^{c}$ and all $z_{I}^{c}$ are tight.

Proof: The verification of the inequality is a straightforward calculation:

$$
\begin{aligned}
& \sum_{i \in I} x_{i}=\sum_{k=1}^{v}\left(\sum_{\left.i \in R_{\delta_{k, m_{k}}} \backslash \bigcup_{\ell \in W_{k} \backslash\left\{m_{k}\right\}}[n] \backslash R_{\delta_{k, \ell}}\right)} x_{i}\right) \\
& =\sum_{k=1}^{v}\left(\sum_{i \in R_{\delta_{k}, m_{k}}} x_{i}-\sum_{\ell \in W_{k} \backslash\left\{m_{k}\right\}} \sum_{i \in[n] \backslash R_{\delta_{k, \ell}}} x_{i}\right) \\
& =\sum_{k=1}^{v}\left(\sum_{i \in R_{\delta_{k}, m_{k}}} x_{i}+\sum_{\ell \in W_{k} \backslash\left\{m_{k}\right\}}\left(-\sum_{i \in[n] \backslash R_{\delta_{k, \ell}}} x_{i}\right)\right) \\
& \geq \sum_{k=1}^{v}\left(\tilde{z}_{R_{\delta_{k, w_{k}}}}^{c}+\sum_{\ell \in W_{k} \backslash\left\{m_{k}\right\}}\left(\tilde{z}_{R_{\delta_{k, \ell}}^{c}}^{c}-z_{[n]}\right)\right) .
\end{aligned}
$$

The second equality holds, since the sets $[n] \backslash R_{\delta_{k, \ell}}$ are for fixed $k$ and varying $\ell$ pairwise disjoint. The last inequality holds, since $\sum_{i \in R_{\delta}} x_{i} \geq \tilde{z}_{R_{\delta}}^{c}$ is equivalent to $-\sum_{i \in[n] \backslash R_{\delta}} x_{i} \geq \tilde{z}_{R_{\delta}}^{c}-z_{[n]}$ for every proper diagonal $\delta$. Since the irredundant facets of the associahedron $A s_{n-1}^{c}$ correspond to inequalities for proper diagonals $\delta$ and these inequalities are tight for $\mathrm{As}_{n-1}^{c}$, the claim follows.

As expected, we have $z_{R_{\delta}}^{c}=\tilde{z}_{R_{\delta}}^{c}$ for all proper diagonals $\delta$, since $v=\left|W_{1}\right|=1$.

\section{Combinatorial description and efficient computation of $y_{I}$}

Proposition 1.2 together with Theorem 2.8 provides a way to compute all Minkowski coefficients $y_{I}$ since all tight values $z_{I}^{c}$ for $A s_{n-1}^{c}$ can be computed. The main goal of this section is to provide the simplified 
formula for $y_{I}$ of Theorem 3.1 which consists of at most four non-zero summand for each $I \subseteq[n]$ and has a combinatorial interpretation.

Throughout this section, we use the following notation and make some general assumptions unless explicitly mentioned otherwise. Let $[n]=\mathrm{D}_{c} \sqcup \mathrm{U}_{c}$ be a partition of $[n]$ induced by a Coxeter element $c$ with $\mathrm{D}_{c}=\left\{d_{1}=1<d_{2}<\cdots<d_{\ell}=n\right\}$ and $\mathrm{U}_{c}=\left\{u_{1}<\cdots<u_{m}\right\}$. Consider a non-empty subset $I \subseteq[n]$ with up and down interval decomposition of type $(v, k)$. If $I$ has a nested up and down interval decomposition, then in particular $v=1$ and $I=(a, b)_{\mathrm{D}_{c}} \cup \bigcup_{i=1}^{k}\left[\alpha_{i}, \beta_{i}\right] \mathrm{U}_{c}$ with $\alpha_{k}<\beta_{k} \leq \alpha_{k+1}$ as before. In this situation, we denote the smallest (respectively largest) element of $I$ by $\gamma$ (respectively $\Gamma$ ) and consider the diagonals

$$
\delta_{1}=\{a, b\}, \quad \delta_{2}=\{a, \Gamma\}, \quad \delta_{3}=\{\gamma, b\}, \quad \text { and } \quad \delta_{4}=\{\gamma, \Gamma\}
$$

The subset of proper diagonals of $\left\{\delta_{1}, \delta_{2}, \delta_{3}, \delta_{4}\right\}$ is denoted by $\mathscr{D}_{I}$. It is convenient to use the mnenomic $\delta$ for diagonals, but unfortunately the meaning of $\delta_{i}$ in this and the preceeding section (starting with Lemma 2.4) is not consistent. We now extend our definition of $R_{\delta}$ and $z_{R_{\delta}}$ to non-proper and degenerate diagonals $\delta$. Is $\delta=\{x, y\}$ not a proper diagonal, we set

$$
R_{\delta}:=\left\{\begin{array}{ll}
\varnothing & \text { if } x, y \in \overline{\mathrm{D}}_{c} \\
{[n]} & \text { otherwise, }
\end{array} \quad \text { and } \quad z_{R_{\delta}}^{c}:= \begin{cases}0 & \text { if } R_{\delta}=\varnothing \\
\frac{n(n+1)}{2} & \text { if } R_{\delta}=[n]\end{cases}\right.
$$

The main result of this section is

Theorem 3.1

Let I be a non-empty subset of $[n]$ with a nested up and down interval decompositionof type $(1, k)$. Then

$$
y_{I}=\sum_{\delta \in \mathscr{D}_{I}}(-1)^{\left|I \backslash R_{\delta}\right|} z_{R_{\delta}}^{c} .
$$

The ideas used to prove Theorem 3.1 also prove the following corollary.

\section{Corollary 3.2}

Let $I$ be a non-empty subset of $[n]$ with a nested up and down interval decompositionof type $(v, k)$ and $v>1$. Then $y_{I}=0$.

The rest of this section sketches the proof of Theorem 3.1. We start with some general remarks and then sketch the proof of Theorem 3.1 if $\mathscr{D}_{I}=\left\{\delta_{1}, \delta_{2}, \delta_{3}, \delta_{4}\right\}$. The same techniques combined with a more detailed statement of Lemma 3.3 settle the remaining cases of Theorem 3.1, see Corollary 3.4

Combining Proposition 1.2 and Theorem 2.8, we obtain

$$
y_{I}=\sum_{J \subseteq I}(-1)^{|I \backslash J|} z_{J}^{c}=\sum_{J \subseteq I}(-1)^{|I \backslash J|} \sum_{i=1}^{v_{J}}\left(\tilde{z}_{R_{\delta_{i, m_{i}^{J}}^{J}}^{c}}+\sum_{j \in W_{i}^{J} \backslash\left\{m_{i}^{J}\right\}}\left(\tilde{z}_{R_{\delta_{i, j}^{J}}^{c}}-z_{[n]}\right)\right) .
$$

This formula is rather awkward at first sight but it turns out that the right-hand side simplifies significantly for our instances $A s_{n-1}^{c}$. We say that a proper diagonal $\delta$ (associated to $J$ ) is of type $\tilde{z}_{R_{\delta}}^{c}$ (in the expression for $y_{I}$ ), if there exists a set $J \subseteq I$ and an index $i \in\left[v_{J}\right]$ such that $\delta=\delta_{i, m_{i}^{J}}^{J}$. Similarly, we say that a proper diagonal $\delta$ (associated to $J$ ) is of type $\left(\tilde{z}_{R_{\delta}}^{c}-z_{[n]}\right)$ (in the expression for $y_{I}$ ), if there exists a set $J$ and indices $i \in\left[v_{J}\right]$ and $j \in W_{i}^{J} \backslash\left\{m_{i}^{J}\right\}$ such that $\delta=\delta_{i, j}^{J}$. 


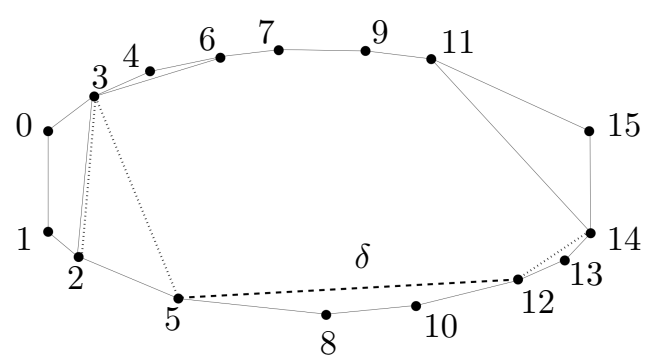

Carsten Lange

Fig. 2: Here $\left.I=(2,14)_{\mathrm{D}_{c}} \cup[3,3]\right]_{\cup_{c}} \cup[6,11] \cup_{c}$ with $\gamma=3$ and $\Gamma=13$. For $\delta=\{5,12\}$, the up and down interval decomposition of $R_{\delta}$ is of type $(1,0)$. Moreover, $\delta$ is associated to $\{8,10\},\{3,8,10\},\{3,8,10,13\}$, and $\{8,10,13\}$ since $(2,5) \cap I=\{3\}$ and $(12,14) \cap I=\{13\}$. Thus the contribution of $\delta$ to $y_{I}$ vanishes. The only diagonals associated to $J \subseteq I$ with up and down interval decomposition of type $(1,0)$ and non-vanishing contribution to $y_{I}$ are diagonals associated to only one subset $J \subseteq I$, i.e. $\delta_{1}=\{2,14\}$ and $\delta_{2}=\{2,13\}$ in this example.

\section{Lemma 3.3}

Let I be a non-empty proper subset of $[n]$ with up and down interval decomposition of type $(1, k)$.

(a) There is no partition $[n]=\mathrm{D}_{c} \sqcup \mathrm{U}_{c}$ induced by a Coxeter element $c$ and no non-empty $I \subset[n]$ such that $\mathscr{D}_{I}$ is one of the following sets:

$$
\varnothing, \quad\left\{\delta_{2}\right\}, \quad\left\{\delta_{3}\right\}, \quad\left\{\delta_{4}\right\}, \quad\left\{\delta_{1}, \delta_{2}\right\}, \quad\left\{\delta_{1}, \delta_{3}\right\}, \quad\left\{\delta_{2}, \delta_{4}\right\}, \quad \text { or } \quad\left\{\delta_{3}, \delta_{4}\right\} .
$$

(b) There is a partition $[n]=\mathrm{D}_{c} \sqcup \mathrm{U}_{c}$ induced by a Coxeter element $c$ and a non-empty $I \subset[n]$ such that $\mathscr{D}_{I}$ is one of the following sets:

$$
\left\{\delta_{1}\right\},\left\{\delta_{1}, \delta_{4}\right\},\left\{\delta_{2}, \delta_{3}\right\},\left\{\delta_{1}, \delta_{2}, \delta_{3}\right\},\left\{\delta_{1}, \delta_{2}, \delta_{4}\right\},\left\{\delta_{1}, \delta_{3}, \delta_{4}\right\},\left\{\delta_{2}, \delta_{3}, \delta_{4}\right\} \text {, or }\left\{\delta_{1}, \delta_{2}, \delta_{3}, \delta_{4}\right\} .
$$

The proof of Part (a) is left to the reader, while a more detailed analysis for Part $(b)$ is implicitly given (and needed) for Corollary 3.4 We now sketch the proof of Theorem 3.1 if $\mathscr{D}_{I}=\left\{\delta_{1}, \delta_{2}, \delta_{3}, \delta_{4}\right\}$.

Proof: We have to analyse the equation for $y_{I}$ given above. Let $\delta$ be some diagonal $\delta_{i, j}^{J}$ that occurs on the equation's right-hand side. In particular, $\delta$ is a proper and non-degenerate diagonal, since $\delta$ is a diagonal of an up and down interval decomposition. By Example 2.3, the up and down interval decomposition of $R_{\delta}$ is either of type $(1,0),(1,1)$ or $(1,2)$. A good understanding which sets $S \subseteq I$ satisfy $\delta \in \mathcal{D}_{S}$ and in which cases $\delta$ associated to $S$ is of type $\tilde{z}_{R_{\delta}}^{c}$ or of type $\left(\tilde{z}_{R_{\delta}}^{c}-z_{[n]}\right)$ in the expression for $y_{I}$ is essential for the simplification. The complete picture is a case study of the mentioned three cases for $R_{\delta}$, two of them split further into subcases. In order to illustrate some of the arguments used, we show the simplest case where $R_{\delta}$ has an up and down interval decomposition of type $(1,0)$.

1. $R_{\delta}$ has up and down decomposition of type $(1,0)$, see Figure 2

Then $R_{\delta}=(\tilde{a}, \tilde{b})_{\mathrm{D}_{c}} \subseteq(a, b)_{\mathrm{D}_{c}}$ and $R_{\delta} \subseteq I$. Let $S \subseteq I$ be a set with $\delta \in \mathcal{D}_{S}$. Then $(\tilde{a}, \tilde{b})_{\mathrm{D}_{c}}$ is a nested component of type $(1,0)$ of $S$ and all other nested components are subsets of $(a, \tilde{a}) \cap I$ and $(\tilde{b}, b) \cap I$. It follows that a set $S$ satisfies $\delta \in \mathcal{D}_{S}$ if and only if

$$
R_{\delta} \subseteq S \subseteq R_{\delta} \cup((a, \tilde{a}) \cap I) \cup((\tilde{b}, b) \cap I) .
$$

We now collect all terms for $\tilde{z}_{R_{\delta}}^{c}$ in the expression for $y_{I}$. Since $\delta$ is a proper diagonal, we have $\tilde{z}_{R_{\delta}}^{c} \neq 0$ and the resulting alternating sum vanishes if and only if there is more than one term of this 
type, that is, if and only if $((a, \tilde{a}) \cap I) \cup((\tilde{b}, b) \cap I) \neq \varnothing$. If $((a, \tilde{a}) \cap I) \cup((\tilde{b}, b) \cap I)=\varnothing$, we obtain $(-1)^{\left|I \backslash R_{\delta}\right|} \tilde{z}_{R_{\delta}}^{c}$ as contribution for $y_{I}$. Moreover, the condition $((a, \tilde{a}) \cap I) \cup((\tilde{b}, b) \cap I)=\varnothing$ guarantees $\delta \in \mathscr{D}_{I}$. The diagonal $\delta_{1}$ is always of this type. Similarly, we have $\delta_{2} \in \mathscr{D}_{I}$ if $\Gamma \in \mathrm{D}_{c}$, $\delta_{3} \in \mathscr{D}_{I}$ if $\gamma \in \mathrm{D}_{c}$, and $\delta_{4} \in \mathscr{D}_{I}$ if $\gamma, \Gamma \in \mathrm{D}_{c}$.

Once the three cases are settled, we have a smaller number of diagonals that have a potential contribution to $y_{I}$. We now proceed by another case study and distinguish the four cases

1. $\gamma, \Gamma \in \mathrm{D}_{c}$,

2. $\gamma \in \mathrm{U}_{c}$ and $\Gamma \in \mathrm{D}_{c}$,

3. $\gamma, \Gamma \in \mathrm{U}_{c}$, and

4. $\gamma \in \mathrm{D}_{c}, \Gamma \in \mathrm{U}_{c}$.

Collecting the diagonals in each of these four cases from the previous analysis, additional terms cancel and the claim follows.

We end this section listing the precise statements needed to settle the degenerate cases $\mathscr{D}_{I} \neq\left\{\delta_{1}, \delta_{2}, \delta_{3}, \delta_{4}\right\}$ of Theorem 3.1 in the following corollary.

\section{Corollary 3.4}

Let I be non-empty proper subset of $[n]$ with up and down interval decomposition of type $(1, k)$.

(a) Suppose that I satisfies one of the following conditions

(i) $\mathscr{D}_{I}=\left\{\delta_{1}\right\}$,

(ii) $\mathscr{D}_{I}=\left\{\delta_{1}, \delta_{3}, \delta_{4}\right\},(a, b)_{\mathrm{D}}=\{\Gamma\}$, and $\gamma \in \mathrm{U}_{c}$

(iii) $\mathscr{D}_{I}=\left\{\delta_{1}, \delta_{2}, \delta_{4}\right\},(a, b)_{\mathrm{D}}=\{\gamma\}$, and $\Gamma \in \mathrm{U}_{c}$

(iv) $\mathscr{D}_{I}=\left\{\delta_{1}, \delta_{2}, \delta_{3}\right\}$ and $(a, b)_{\mathrm{D}}=\{\gamma, \Gamma\}$, or

(v) $\mathscr{D}_{I}=\left\{\delta_{2}, \delta_{3}, \delta_{4}\right\}$ and $(a, b)_{\mathrm{D}}=\varnothing$.

Then the Minkowski coefficient $y_{I}$ of $\mathrm{As}_{n-1}^{c}$ is

$$
y_{I}=\sum_{\delta \in \mathscr{D}_{I}}(-1)^{\left|I \backslash R_{\delta}\right|} z_{R_{\delta_{1}}} .
$$

(b) Suppose that I satisfies one of the following conditions

(i) $\mathscr{D}_{I}=\left\{\delta_{1}, \delta_{4}\right\}$,

(ii) $\mathscr{D}_{I}=\left\{\delta_{2}, \delta_{3}\right\}$,

(iii) $\mathscr{D}_{I}=\left\{\delta_{1}, \delta_{3}, \delta_{4}\right\}$ and $\bigcup_{i=1}^{k}\left[\alpha_{i}, \beta_{i}\right] \mathrm{U}_{c}=\{\Gamma\}$,

(iv) $\mathscr{D}_{I}=\left\{\delta_{1}, \delta_{2}, \delta_{4}\right\}$ and $\bigcup_{i=1}^{k}\left[\alpha_{i}, \beta_{i}\right]_{\mathrm{U}_{c}}=\{\gamma\}$,

(v) $\mathscr{D}_{I}=\left\{\delta_{1}, \delta_{2}, \delta_{3}\right\}$ and $\bigcup_{i=1}^{k}\left[\alpha_{i}, \beta_{i}\right]_{\mathrm{U}_{c}}=\{\gamma, \Gamma\}$.

Then the Minkowski coefficient $y_{I}$ of $\mathrm{As}_{n-1}^{c}$ is

$$
y_{I}=(-1)^{|\{\gamma, \Gamma\}|} z_{[n]}+\sum_{\delta \in \mathscr{D}_{I}}(-1)^{\left|I \backslash R_{\delta}\right|} z_{R_{\delta_{1}}} .
$$

\section{References}

[ABD10] Federico Ardila, Carolina Benedetti, and Jeffrey Doker. Matroid polytopes and their volumes. Discrete Comput Geom, 43:841-854, 2010. 
[CFZ02] Frédéric Chapoton, Sergey Fomin, and Andrei Zelevinksy. Polytopal realizations of generalized associahedra. Canad Math Bull, 45:537-566, 2002.

[FZ03] Sergey Fomin and Andrei Zelevinksy. Y-systems and generalized associahedra. Ann. of Math., 158:977-1018, 2003.

[FZ07] Sergey Fomin and Andrei Zelevinksy. Cluster algebras iv. coefficients. Compos Math, 143:112-164, 2007.

[HL07] Christophe Hohlweg and Carsten Lange. Realizations of the associahedron and cyclohedron. Discrete Comput Geom, 37:517-543, 2007.

[HLT11] Christophe Hohlweg, Carsten Lange, and Hugh Thomas. Permutahedra and generalized associahedra. Adv Math, 226:608-640, 2011.

[Kal88] Gil Kalai. A simple way to tell a simple polytope from its graph. J. Combin Theory Ser A, 49:381-383, 1988.

[Lod04] Jean-Louis Loday. Realizations of the Stasheff polytope. Arch Math, 83:267-278, 2004.

[Pos09] Alexander Postnikov. Permutahedra, associahedra, and beyond. Int Math Res Not, pages 10261106, 2009.

[RS09] Nathan Reading and David Speyer. Cambrian fans. J Europ Math Soc, 11:411-447, 2009.

[RSS03] Günter Rote, Francisco Santos, and Ileana Streinu. Discrete and Computational Geometry - The Goodman-Pollack Festschrift, chapter Expansive Motions and the Polytope of Pointed Pseudo-Triangulations, pages 699-736. Number 25 in Algorithms and Combinatorics. Springer-Verlag, Berlin, 2003.

[YZ08] Shih-Wei Yang and Andrei Zelevinksy. Cluster algebras of finite type via coxeter elements and principal minors. Transform Groups, 13:855-895, 2008.

[Zie98] Günter M. Ziegler. Lectures on Polytopes, volume 152 of Graduate Texts in Mathematics. Springer-Verlag, Heidelberg, 1998. 